\title{
Special Associated Curves in Euclidean 3-Space
}

\author{
Sezai KIZILTUД, ${ }^{1}$ and Gokhan MUMCU² \\ ${ }^{1}$ Erzincan University \\ ${ }^{2}$ Erzincan Universitesi Fen Edebiyat Fakultesi
}

April 28, 2020

\begin{abstract}
First, we study a new tip of unit speed associated curves in the E3 like a normal-direction curve and normal-donor curve. Then we achieve qualification for these curves. Moreover, we confer applications of normal-direction to some special curves such as helix, slant helix, plane curve or normal-direction (ND)-normal curves in E3. And, we show that slant helices and rectifying curves might be assemble by using normal-direction curves.
\end{abstract}

\begin{abstract}
First, we study a new tip of unit speed associated curves in the $E^{3}$ like a normal-direction curve and normal-donor curve. Then we achieve qualification for these curves. Moreover, we confer applications of normal-direction to some special curves such as helix, slant helix, plane curve or normal-direction $(N D$ )-normal curves in $E^{3}$. And, we show that slant helices and rectifying curves might be assemble by using normal-direction curves.
\end{abstract}

MSC: 53A04.

Key words: Incorporated curve; normal-direction curve; normal-donor curve.

\section{Introduction}

In the curve theory of Euclidean space, the momentous question is achieve a characterization in order to a regular curve. The specification may be dedicated for a single curve or for a curve pair. Helix, slant helix, plane curve, spherical curve, etc. are well-known instance of single special curves $[1,9,10,13,18]$ and these curves, exclusively the helices, are used in many applications $[2,7,8,15]$. Additionally, special curves can be defined by careful Frenet planes. Providing the position vector of a curve always lies on its rectifying, osculating or normal planes, then the curve is called rectifying curve, osculating curve or normal curve, seriatim [4]. Exclusively, therein obtain a basic correlation among rectifying curves and Darboux vectors, which trick some momentous parts in mechanics, kinematics as well as in differential geometry in describing the curves of constant motion [5,12].

Besides, special curve pairs are characterized by some relationships between their Frenet vectors or curvatures. Involute-evolute curves, Bertrand curves, Mannheim curves are admitted sample of curve pairs and studious by some mathematicians $[3,11-13,16,17]$.

Hereabout, a new curve pair in the Euclidean 3 -space $E^{3}$ has been defined by Choi and Kim [6]. They have considered an integral curve $\gamma$ of a unit vector field $X$ defined in the Frenet basis of a Frenet curve $\alpha$ and they have given the definitions and characterizations of principal-directional curve and principal-donor curve in $E^{3}$.They have also given some applications of these curves to some special curves. 
In the current paper, we consider a new type of associated curve and define a new curve pair such as normaldirection curve and normal-donor curve in $E^{3}$. We obtain some characterizations for these curves and show that normal-direction curve is an evolute of normal-donor curve. Moreover, we give some applications of normal-direction curve to some special curves such as helix, slant helix or plane curve.

\section{Preliminaries}

This section includes a brief summary of space curves and definitions of general helix and slant helix in the Euclidean 3-space $E^{3}$.

A unit speed curve $\alpha: I \rightarrow E^{3}$ is called a general helix if there is a constant vector $u$, so that $\langle T, u\rangle=\cos \theta$ is constant along the curve, where $\theta \neq \pi / 2$ and $T(s)=\alpha^{\prime}(s)$ is unit tangent vector of $\alpha$ at $s$. The curvature (or first curvature) of $\alpha$ is defined by $\kappa(s)=\left\|\alpha^{\prime \prime}(s)\right\|$. Then, the curve $\alpha$ is called Frenet curve, if $\kappa(s) \neq 0$, and the unit principal normal vector $N(s)$ of the curve $\alpha$ at $s$ is given by $\alpha^{\prime \prime}(s)=\kappa(s) N(s)$. The unit vector $B(s)=T(s) \times N(s)$ is called the unit binormal vector of $\alpha$ at $s$. Then $\{T, N, B\}$ is called the Frenet frame of $\alpha$. For the derivatives of the Frenet frame, the following Frenet-Serret formulae hold:

$$
\begin{aligned}
& T^{\prime} \\
& N^{\prime}=\text { [ } \\
& B^{\prime} \\
& \begin{array}{lll}
0 & \kappa & 0
\end{array} \\
& \begin{array}{llll}
-\kappa & 0 & \tau
\end{array} \\
& \begin{array}{lll}
0 & -\tau & 0
\end{array}
\end{aligned}
$$

where $\tau(s)$ is the torsion (or second curvature) of $\alpha$ at $s$. It is well-known that the curve $\alpha$ is a general helix if and only if $\frac{\tau}{\kappa}(s)=$ constant [17]. If both $\kappa(s) \neq 0$ and $\tau(s)$ are constants, we call $\alpha$ as a circular helix. A curve $\alpha$ with $\kappa(s) \neq 0$ is called a slant helix if the principal normal lines of $\alpha$ make a constant angle with a fixed direction. Also, a slant helix $\alpha$ in $E^{3}$ is characterized by the differential equation of its curvature $\kappa$ and its torsion $\tau$ given by

$$
\frac{\kappa^{2}}{\left(\kappa^{2}+\tau^{2}\right)^{3 / 2}}\left(\frac{\tau}{\kappa}\right)^{\prime}=\text { constant }
$$

(See [11]).

Now, we give the definitions of some associated curves defined by Choi and Kim [6]. Let $I \subset \mathbb{R}$ be an open interval. For a Frenet curve $\phi: I \rightarrow E^{3}$, consider a vector field $X$ given by

$$
\chi(s)=\vartheta(s) \mathbf{T}(\mathbf{s})+v(s) \mathbf{N}(\mathbf{s})+\omega(s) \mathbf{B}(\mathbf{s})
$$


where $\vartheta, v$ and $\omega$ are arbitrary differentiable functions of $s$ which is the arc length parameter of $\phi$. Let

$$
\vartheta^{2}(s)+v^{2}(s)+\omega^{2}(s)=1
$$

holds. Then the definitions of $\chi$-direction curve and $\chi$-donor curve in $E^{3}$ are given as follows.

Definition 2.1. ([6]) Let $\phi$ be a Frenet curve in Euclidean 3-space $E^{3}$ and $\chi$ be a unit vector field satisfying the equations (2) and (3). The integral curve $\delta: I \rightarrow E^{3}$ of $\chi$ is called an $\chi$-direction curve of $\phi$. The curve $\phi$ whose $\chi$-direction curve is $\delta$ is called the $\phi$-donor curve of $\delta$ in $E^{3}$.

Definition 2.2. ([6]) An integral curve of principal normal vector $\mathbf{N}(\mathrm{s})$ (resp. binormal vector $\mathbf{B}(\mathrm{s})$ ) of $\phi$ in (2) is called the principal-direction curve (resp. binormal-direction curve) of $\phi$ in $E^{3}$.

Remark 2.1. ([6]) A principal-direction (resp. the binormal-direction) curve is an integral curve of $\phi(s)$ with $\vartheta(s)=\omega(s)=0, v(s)=1($ resp. $\vartheta(s)=v(s)=0, \omega(s)=1)$ for all $s$ in $(2)$.

\section{Normal-direction curve and normal-donor curve in $E^{3}$}

In this section, we will give definitions of normal-direction curve and normal donor curve in $E^{3}$. We obtain some theorems and results characterizing these curves. First, we give the following definition.

Definition 3.1. Let $\alpha$ be a Frenet curve in $E^{3}$ and $X$ be a unit vector field lying on the normal plane of $\alpha$ and defined by

$$
X(s)=v(s) N(s)+w(s) B(s), v(s) \neq 0, w(s) \neq 0
$$

and satisfying that the vectors $X^{\prime}(s)$ and $T(s)$ are linearly dependent. The integral curve $\gamma: I \rightarrow E^{3}$ of $X(s)$ is called a normal-direction curve of $\alpha$. The curve $\alpha$ whose normal -direction curve is $\gamma$ is called the normal-donor curve in $E^{3}$.

The Frenet frame is a rotation-minimizing with respect to the principal normal $N[9]$. If we consider a new frame given by $\{T, X, M\}$ where $M=T \times X$, we have that this new frame is rotation-minimizing with respect to $T$, i.e., the unit vector $X$ belongs to a rotation-minimizing frame.

Since, $X(s)$ is a unit vector and $\gamma: I \rightarrow E^{3}$ is an integral curve of $X(s)$, without loss of generality we can take $s$ as the arc length parameter of $\gamma$ and we can give the following characterizations in the view of these information. 
Theorem 3.1. Let $\alpha: I \rightarrow E^{3}$ be a Frenet curve and an integral curve of $X(s)=v(s) N(s)+w(s) B(s)$ be the curve $\gamma: I \rightarrow E^{3}$. Then, $\gamma$ is a normal-direction curve of $\alpha$ if and only if the following equalities hold,

$$
v(s)=\sin \left(\int \tau d s\right) \neq 0, \quad w(s)=\cos \left(\int \tau d s\right) \neq 0 .
$$

Proof: Since $\gamma$ is a normal-direction curve of $\alpha$, from Definition 3.1, we have

$$
X(s)=v(s) N(s)+w(s) B(s)
$$

and

$$
v^{2}(s)+w^{2}(s)=1
$$

Differentiating (6) with respect to $s$ and by using the Frenet formulas, it follows

$$
X^{\prime}(s)=-v \kappa T+\left(v^{\prime}-w \tau\right) N+\left(w^{\prime}+v \tau\right) B
$$

Since we have that $X^{\prime}$ and $T$ are linearly dependent. Then from (8) we can write

$$
\begin{aligned}
& -v \kappa \neq 0, \\
& v^{\prime}-w \tau=0, \\
& w^{\prime}+v \tau=0 .
\end{aligned}
$$

(9)

\section{\{}

The solutions of second and third differential equations are

$$
v(s)=\sin \left(\int \tau d s\right) \neq 0, \quad w(s)=\cos \left(\int \tau d s\right) \neq 0,
$$


respectively, which completes the proof.

Theorem 3.2. Let $\alpha: I \rightarrow E^{3}$ be a Frenet curve. If $\gamma$ is the normal-direction curve of $\alpha$, then $\gamma$ is a space evolute of $\alpha$.

Proof: Since $\gamma$ is an integral curve of $X$, we have $\gamma^{\prime}=X$. Denote the Frenet frame of $\gamma$ by $\{\bar{T}, \bar{N}, \bar{B}\}$. Differentiating $\gamma^{\prime}=X$ with respect to $s$ and by using Frenet formulas we get

$$
X^{\prime}=\bar{T}^{\prime}=\bar{\kappa} \bar{N}
$$

Furthermore, we know that $X^{\prime}$ and $T$ are linearly dependent. Then from (10) we get $\bar{N}$ and $T$ are linearly dependent, i.e, $\gamma$ is a space evolute of $\alpha$.

Theorem 3.3. Let $\alpha: I \rightarrow E^{3}$ be a Frenet curve. If $\gamma$ is the normal direction curve of $\alpha$, then the curvature $\bar{\kappa}$ and the torsion $\bar{\tau}$ of $\gamma$ are given as follows,

$$
\bar{\kappa}=\kappa\left|\sin \left(\int \tau d s\right)\right|, \bar{\tau}=\kappa \cos \left(\int \tau d s\right) .
$$

Proof: From (8), (9) and (10), we have

$$
\bar{\kappa} \bar{N}=-v \kappa T
$$

By considering (12) and (5) we obtain

$$
\bar{\kappa} \bar{N}=-\kappa \sin \left(\int \tau d s\right) T
$$

which gives us

$$
\bar{\kappa}=\kappa\left|\sin \left(\int \tau d s\right)\right|
$$


Moreover, from (13) and (14), we can write

$$
\bar{N}=T \text {. }
$$

Then, we have

$$
\bar{B}=\bar{T} \times \bar{N}=\cos \left(\int \tau d s\right) N-\sin \left(\int \tau d s\right) B .
$$

Differentiating (16) with respect to $s$ gives

$$
\bar{B}^{\prime}=-\kappa \cos \left(\int \tau d s\right) T
$$

Since $\bar{\tau}=-\left\langle\bar{B}^{\prime}, \bar{N}\right\rangle=-\left\langle\bar{B}^{\prime}, T\right\rangle$, from (17) it follows

$$
\bar{\tau}=\kappa \cos \left(\int \tau d s\right)
$$

that finishes the proof.

Corollary 3.1. Let $\gamma$ be a normal-direction curve of the curve $\alpha$. Then the relationships between the Frenet frames of curves are given as follows,

$$
X=\bar{T}=\sin \left(\int \tau d s\right) N+\cos \left(\int \tau d s\right) B, \bar{N}=T, \bar{B}=\cos \left(\int \tau d s\right) N-\sin \left(\int \tau d s\right) B
$$

Proof: The proof is clear from Theorem 3.3.

Theorem 3.4. Let $\gamma$ be a normal-direction curve of $\alpha$ with curvature $\bar{\kappa}$ and torsion $\bar{\tau}$. Then curvature $\kappa$ and torsion $\tau$ of $\alpha$ are given by

$$
\kappa=\sqrt{\bar{\kappa}^{2}+\bar{\tau}^{2}}, \tau=\frac{\bar{\tau}^{2}}{\bar{\kappa}^{2}+\bar{\tau}^{2}}\left(\frac{\bar{\kappa}}{\bar{\tau}}\right)^{\prime}
$$


Proof: From (14) and (18), we easily get

$$
\kappa=\sqrt{\bar{\kappa}^{2}+\bar{\tau}^{2}} .
$$

Substituting (19) into (14) and (18), it follows

$$
\left|\sin \left(\int \tau d s\right)\right|=\frac{\bar{\kappa}}{\sqrt{\bar{\kappa}^{2}+\bar{\tau}^{2}}},
$$

$$
\cos \left(\int \tau d s\right)=\frac{\bar{\tau}}{\sqrt{\bar{\kappa}^{2}+\bar{\tau}^{2}}},
$$

respectively. Differentiating (20) with respect to $s$, we have

$$
\tau \cos \left(\int \tau d s\right)=\frac{\bar{\tau}\left(\bar{\kappa}^{\prime} \bar{\tau}-\bar{\kappa} \bar{\tau}^{\prime}\right)}{\left(\bar{\kappa}^{2}+\bar{\tau}^{2}\right)^{3 / 2}} .
$$

From (21) and (22), it follows

$$
\tau=\frac{\bar{\kappa}^{\prime} \bar{\tau}-\bar{\kappa} \bar{\tau}^{\prime}}{\bar{\kappa}^{2}+\bar{\tau}^{2}}
$$

or equivalently,

$$
\tau=\frac{\bar{\tau}^{2}}{\bar{\kappa}^{2}+\bar{\tau}^{2}}\left(\frac{\bar{\kappa}}{\bar{\tau}}\right)^{\prime}
$$


Theorem 3.4 leads us to give the following corollary whose proof is clear.

Corollary 3.2. Let $\gamma$ with the curvature $\bar{\kappa}$ and the torsion $\bar{\tau}$ be a normal-direction curve of $\alpha$. Then

$$
\frac{\tau}{\kappa}=-\frac{\bar{\kappa}^{2}}{\left(\bar{\kappa}^{2}+\bar{\tau}^{2}\right)^{3 / 2}}\left(\frac{\bar{\tau}}{\bar{\kappa}}\right)^{\prime}
$$

is satisfied, where $\kappa$ and $\tau$ are curvature and torsion of $\alpha$, respectively.

\section{Applications of normal-direction curves}

In this section, we focus on relations between normal-direction curves and some special curves such as general helix, slant helix, plane curve or rectifying curve in $E^{3}$.

\subsection{General helices, slant helices and plane curves}

Considering Corollary 3.2, we have the following theorems which gives a way to construct the examples of slant helices by using general helices.

Theorem 4.1. Let $\alpha: I \rightarrow E^{3}$ be a Frenet curve in $E^{3}$ and $\gamma$ be a normal-direction curve of $\alpha$. Then the followings are equivalent,

i) A Frenet curve $\alpha$ is a general helix in $E^{3}$.

ii) $\alpha$ is a normal-donor curve of a slant helix.

iii) A normal-direction curve of $\alpha$ is a slant helix.

Theorem 4.2. Let $\alpha: I \rightarrow E^{3}$ be a Frenet curve in $E^{3}$ and $\gamma$ be a normal-direction curve of $\alpha$. Then the followings are equivalent,

i) A Frenet curve $\alpha$ is a plane curve in $E^{3}$.

ii) $\alpha$ is a normal-donor curve of a general helix.

iii) A normal-direction curve of $\alpha$ is a general helix.

Example 4.1. Let consider the general helix given by the parametrization $\alpha(s)=\left(\cos \frac{s}{\sqrt{2}}, \sin \frac{s}{\sqrt{2}}, \frac{s}{\sqrt{2}}\right)$ in $E^{3}$ (Fig. 1). The Frenet vectors and curvatures of $\alpha$ are obtained as follows,

$$
\begin{gathered}
T(s)=\left(-\frac{1}{\sqrt{2}} \sin \frac{s}{\sqrt{2}}, \frac{1}{\sqrt{2}} \cos \frac{s}{\sqrt{2}}, \frac{1}{\sqrt{2}}\right), \\
N(s)=\left(-\cos \frac{s}{\sqrt{2}}, \sin \frac{s}{\sqrt{2}}, 0\right)
\end{gathered}
$$




$$
\begin{gathered}
B(s)=\left(\frac{1}{\sqrt{2}} \sin \frac{s}{\sqrt{2}},-\frac{1}{\sqrt{2}} \cos \frac{s}{\sqrt{2}}, \frac{1}{\sqrt{2}}\right), \\
\kappa=\tau=\frac{1}{2} .
\end{gathered}
$$

Then we have $X(s)=\left(x_{1}(s), x_{2}(s), x_{3}(s)\right)$ where

$$
\begin{aligned}
& x_{1}(s)=-\sin \left(\frac{s}{2}+c\right) \cos \frac{s}{\sqrt{2}}+\frac{1}{\sqrt{2}} \cos \left(\frac{s}{2}+c\right) \sin \frac{s}{\sqrt{2}} \\
& x_{2}(s)=\sin \left(\frac{s}{2}+c\right) \sin \frac{s}{\sqrt{2}}-\frac{1}{\sqrt{2}} \cos \left(\frac{s}{2}+c\right) \cos \frac{s}{\sqrt{2}} \\
& x_{3}(s)=\frac{1}{\sqrt{2}} \cos \left(\frac{s}{2}+c\right) .
\end{aligned}
$$

and $c$ is integration constant. Now, we can construct a slant helix $\gamma$ which is also a normal-direction curve of $\alpha$ (Fig. 2):

$$
\gamma=\int_{0}^{s} \gamma^{\prime}(s) d s=\int_{0}^{s} X(s) d s=\left(\gamma_{1}(s), \gamma_{2}(s), \gamma_{3}(s)\right)
$$

where

$$
\begin{aligned}
& \gamma_{1}(s)=\int_{0}^{s}\left[-\sin \left(\frac{s}{2}+c\right) \cos \frac{s}{\sqrt{2}}+\frac{1}{\sqrt{2}} \cos \left(\frac{s}{2}+c\right) \sin \frac{s}{\sqrt{2}}\right] d s \\
& \gamma_{2}(s)=\int_{0}^{s}\left[\sin \left(\frac{s}{2}+c\right) \sin \frac{s}{\sqrt{2}}-\frac{1}{\sqrt{2}} \cos \left(\frac{s}{2}+c\right) \cos \frac{s}{\sqrt{2}}\right] d s \\
& \gamma_{3}(s)=\int_{0}^{s} \frac{1}{\sqrt{2}} \cos \left(\frac{s}{2}+c\right) d s
\end{aligned}
$$

[ width $=3 \mathrm{in}$, height $=3 \mathrm{in}$, keepaspectratio, $] 1$ width $=2.96 \mathrm{in}$, height $=2.96 \mathrm{in}$, keepaspectratio $=$ false $] 2$

Fig. 1. General helix $\alpha$. Fig. 2. Slant helix $\gamma$ constructed by $\alpha$.

\subsection{ND-normal Curves}

In this subsection we define normal-direction $(N D)$-normal curves in $E^{3}$ and give the relationships between normal-direction curves and $N D$-normal curves.

A space curve whose position vector always lies in its normal plane is called normal curve [5]. Moreover, if the Frenet frame and curvatures of a space curve are given by $\{T, N, B\}$ and $\kappa, \tau$, respectively, then the vector $\tilde{D}(s)=\frac{\tau}{\kappa}(s) T(s)+B(s)$ is called modified Darboux vector of the curve [11]. 
Let now $\alpha$ be a Frenet curve with Frenet frame $\{T, N, B\}$ and $\gamma$ a normal-direction curve of $\alpha$. The curve $\gamma$ is called normal-direction normal curve (or $N D$-normal curve) of $\alpha$, if the position vector of $\gamma$ always lies on the normal plane of its normal-donor curve $\alpha$.

The definition of $N D$-normal curve allows us to write the following equality,

$$
\gamma(s)=m(s) N(s)+n(s) B(s)
$$

where $m(s), n(s)$ are non-zero differentiable functions of $s$. Since $\gamma$ is normal-direction curve of $\alpha$, from Corollary 3.1, we have

$$
\begin{aligned}
& N=\sin \left(\int \tau d s\right) \bar{T}+\cos \left(\int \tau d s\right) \bar{B}, \\
& B=\cos \left(\int \tau d s\right) \bar{T}-\sin \left(\int \tau d s\right) \bar{B} .
\end{aligned}
$$

Substituting (26) in (25) gives

$$
\gamma(s)=\left[m \sin \left(\int \tau d s\right)+n \cos \left(\int \tau d s\right)\right] \bar{T}+\left[m \cos \left(\int \tau d s\right)-n \sin \left(\int \tau d s\right)\right] \bar{B} .
$$

Writing

$\rho(s)=m \sin \left(\int \tau d s\right)+n \cos \left(\int \tau d s\right)$,

$\sigma(s)=m \cos \left(\int \tau d s\right)-n \sin \left(\int \tau d s\right)$,

$(28)$

in (27) and differentiating the obtained equality we obtain

$$
\bar{T}=\rho^{\prime} \bar{T}+(\rho \bar{\kappa}-\sigma \bar{\tau}) \bar{N}+\sigma^{\prime} \bar{B} .
$$


Then we have

$$
\sigma=a=\text { constant }, \rho=s+b=\frac{\bar{\tau}}{\bar{\kappa}} a,
$$

where $a, b$ are non-zero integration constants. From (30), it follows that

$$
\gamma(s)=a\left(\frac{\bar{\tau}}{\bar{\kappa}} \bar{T}+\bar{B}\right)(s)=a \tilde{\bar{D}}(s)
$$

where $\tilde{\bar{D}}$ is the modified Darboux vector of $\gamma$.

Now we can give the followings which characterize $N D$-normal curves.

Theorem 4.3. Let $\alpha: I \rightarrow E^{3}$ be a Frenet curve in $E^{3}$ and $\gamma$ be a normal-direction curve of $\alpha$. If $\gamma$ is a $N D$-normal curve in $E^{3}$, then we have the followings,

i) $\gamma$ is a rectifying curve in $E^{3}$ whose curvatures satisfy $\frac{\bar{\tau}}{\bar{\kappa}}=\frac{s+b}{a}$ where $a, b$ are non-zero constants.

ii) The position vector and modified Darboux vector $\tilde{\bar{D}}$ of $\gamma$ are linearly dependent.

Theorem 4.3 gives a way to construct a rectifying curve by using normal-donor curve as follows:

Corollary 4.1. Let $\alpha: I \rightarrow E^{3}$ be a Frenet curve in $E^{3}$ and $\gamma$ a ND-normal curve of $\alpha$ in $E^{3}$. Then the position vector of $\gamma$ is obtained as follows,

$$
\gamma(s)=\left[(s+b) \sin \left(\int \tau d s\right)+a \cos \left(\int \tau d s\right)\right] N(s)+\left[(s+b) \cos \left(\int \tau d s\right)-a \sin \left(\int \tau d s\right)\right] B(s)
$$

where $a, b$ are non-zero integration constants.

Proof. The proof is clear from (25), (28) and (30).

Example 4.2. Let consider the general helix given by the parametrization

$$
\alpha(s)=\left(\sqrt{1+s^{2}}, s, \ln \left(s+\sqrt{1+s^{2}}\right)\right)
$$

and drawn in Fig. 3. Frenet vectors and curvatures of the curve are 


$$
\begin{gathered}
T(s)=\frac{1}{\sqrt{2} \sqrt{1+s^{2}}}\left(s, \sqrt{1+s^{2}}, 1\right), \\
N(s)=\frac{1}{\sqrt{1+s^{2}}}(1,0,-s), \\
B(s)=\frac{1}{\sqrt{2} \sqrt{1+s^{2}}}\left(-s, \sqrt{1+s^{2}},-1\right), \\
\kappa=\tau=\frac{1+s^{2}}{2},
\end{gathered}
$$

respectively. Then from Corollary 4.1, a $N D$-normal curve $\gamma$ is obtained as follows,

$$
\begin{aligned}
\gamma(s)= & \left(\frac{1}{\sqrt{1+s^{2}}}\left[(s+b) \sin \left(\frac{s}{2}+\frac{s^{3}}{6}+c\right)+a \cos \left(\frac{s}{2}+\frac{s^{3}}{6}+c\right)\right]\right. \\
& -\frac{s}{\sqrt{2\left(1+s^{2}\right)}}\left[(s+b) \cos \left(\frac{s}{2}+\frac{s^{3}}{6}+c\right)-a \sin \left(\frac{s}{2}+\frac{s^{3}}{6}+c\right)\right], \\
& -\frac{1}{\sqrt{2}}\left[(s+b) \cos \left(\frac{s}{2}+\frac{s^{3}}{6}+c\right)-a \sin \left(\frac{s}{2}+\frac{s^{3}}{6}+c\right)\right] \\
& -\frac{s}{\sqrt{1+s^{2}}}\left[(s+b) \sin \left(\frac{s}{2}+\frac{s^{3}}{6}+c\right)+a \cos \left(\frac{s}{2}+\frac{s^{3}}{6}+c\right)\right] \\
& \left.-\frac{1}{\sqrt{2\left(1+s^{2}\right)}}\left[(s+b) \cos \left(\frac{s}{2}+\frac{s^{3}}{6}+c\right)-a \sin \left(\frac{s}{2}+\frac{s^{3}}{6}+c\right)\right]\right)
\end{aligned}
$$

which is also a rectifying curve in the view of Theroem 4.3 and drawn in Figures $4,5,6$ by choosing $a=b=$ $1, c=0$.

$[$ width $=2.92 \mathrm{in}$, height $=2.92 \mathrm{in}$, keepaspectratio $=$ false $] 3$ [width $=2.92 \mathrm{in}$, height $=2.92 \mathrm{in}$, keepaspectratio $=$ false $] 4$

Fig. 3. General helix $\alpha$. Fig 4. $N D$-normal curve $\gamma$ for $-\pi \leq s \leq \pi$.

$[$ width $=3.00 \mathrm{in}$, height $=3.00 \mathrm{in}$, keepaspectratio $=$ false $] 5$ width $=3.00 \mathrm{in}$, height $=3.00 \mathrm{in}$, keepaspectratio $=$ false 6

Fig 5. $N D$-normal curve $\gamma$ for $\frac{-3 \pi}{2} \leq s \leq \frac{3 \pi}{2}$. Fig 6. $\quad N D$-normal curve $\gamma$ for $-2 \pi \leq s \leq 2 \pi$. 


\section{References}

[1] Barros, M., General helices and a theorem of Lancret, Proc. Amer. Math. Soc., 125 (5) (1997) 1503-1509.

[2] Beltran, J.V., Monterde, J., A characterization of quintic helices, J. Comput. Appl. Math., 206 (1) (2007) $116-121$.

[3] Burke, J.F., Bertrand curves associated with a pair of curves. Mathematics Magazine, 34(1) (1960) 60-62.

[4] Chen, B.Y., When does the position vector of a space curve always lie in its normal plane?, Amer. Math. Monthly, 110 (2003) 147-152.

[5] Chen, B.Y., Dillen, F., Rectifying curves as centrodes and extremal curves, Bull. Inst. Math. Academia Sinica, 33 (2005) 77-90.

[6] Choi, J.H., Kim, Y.H., Associated curves of a Frenet curve and their applications, Applied Mathematics and Computation, 218 (2012) 9116-9124.

[7] Da Fonseca, A.F., Malta, C.P., Lancret helices, arXiv:physics/0507105v1 [physics.bio-ph].

[8] Farouki, R.T., Han, C.Y., Manni, C., Sestini, A., Characterization and construction of helical polynomial space curves, J. Comput. Appl. Math., 162 (2) (2004) 365-392.

[9] Farouki, R.T., Giannelli, G., Sampoli, M.C., Sestini, A., Rotation-minimizing osculating frames, Computer Aided Geometric Design, 31 (2014) 27-42.

[10] Hayden, H.A., On a generalized helix in a Riemannian $n$-space, Proc. Lond. Math. Soc., 32 (1931) 337-345.

[11] Izumiya, S., Takeuchi, N., New special curves and developable surfaces, Turk. J. Math., 28 (2004) 153-163.

[12] Kahraman, T., Önder, M., Kazaz, M., Uğurlu, H.H., Some Characterizations of Mannheim Partner Curves in Minkowski 3-space, Proceedings of the Estonian Academy of Sciences, 60(4) (2011) 210-220.

[13] Hanif, M., Önder, M., Generalized quaternionic involute evolute curves in the Euclidean four-space E4, Mathematical Methods in the Applied Sciences, (2020).

[14] Otsuki, T., Differential Geometry, Asakura Publishing Co. Ltd., Tokyo, (in Japanese), (1961).

[15] Puig-Pey, J. Gálvez, A., Iglesias, A., Helical curves on surfaces for computer-aided geometric design and manufacturing, in: Computational Science and its Applications-ICCSA 2004. Part II, 771-778, in: Lecture Notes in Comput. Sci., vol. 3044, Springer, Berlin, (2004).

[16] Struik, D.J., Lectures on Classical Differential Geometry, $2^{\text {nd }}$ ed. Addison Wesley, Dover, (1988).

[17] Wang, F., Liu, H., Mannheim partner curves in 3-Euclidean space, Mathematics in Practice and Theory, 37(1) (2007) 141-143.

[18] Whittemore, J.K., Bertrand curves and helices. Duke Math. J., 6(1) (1940) 235-245. 